\title{
Inflammatory Activation of Microglia and Astrocytes in Manganese Neurotoxicity
}

\author{
Ronald B. Tjalkens, \\ Program in Cell and Molecular Biology, Colorado State University, Fort Collins, CO 80523-1680, \\ USA \\ Professor of Toxicology and Neuroscience, Center for Environmental Medicine, Colorado State \\ University, Fort Collins, CO 80523-1680, USA \\ Department of Environmental and Radiological Health Sciences, Colorado State University, Fort \\ Collins, CO 80523-1680, USA
}

Katriana A. Popichak, and

Program in Cell and Molecular Biology, Colorado State University, Fort Collins, CO 80523-1680, USA

\section{Kelly A. Kirkley}

Professor of Toxicology and Neuroscience, Center for Environmental Medicine, Colorado State University, Fort Collins, CO 80523-1680, USA

Department of Environmental and Radiological Health Sciences, Colorado State University, Fort Collins, CO 80523-1680, USA

\section{Abstract}

Neurotoxicity due to excessive exposure to manganese (Mn) has been described as early as 1837 (Couper, Br Ann Med Pharm Vital Stat Gen Sci 1:41-42, 1837). Extensive research over the past two decades has revealed that Mn-induced neurological injury involves complex pathophysiological signaling mechanisms between neurons and glial cells. Glial cells are an important target of $\mathrm{Mn}$ in the brain, both for sequestration of the metal, as well as for activating inflammatory signaling pathways that damage neurons through overproduction of numerous reactive oxygen and nitrogen species and inflammatory cytokines. Understanding how these pathways are regulated in glial cells during Mn exposure is critical to determining the mechanisms underlying permanent neurological dysfunction stemming from excess exposure. The subject of this review will be to delineate mechanisms by which Mn interacts with glial cells to perturb neuronal function, with a particular emphasis on neuroinflammation and neuroinflammatory signaling between distinct populations of glial cells.

\section{Keywords}

Manganism; Pattern recognition receptors (PRRs); Astrogliosis; Glial fibrillary acidic protein (GFAP); Parkinson's disease (PD) 


\section{Introduction}

Glia represent a diverse class of cells grouped together due their status as non-excitable neural cells that lack the ability to form an action potential and thus transmit electrical signals. Within the central nervous system (CNS), glia represent $90 \%$ of all cells and are classified on the basis of morphology, function, and location consisting of astrocytes, microglia, oligodendrocytes, and ependymal cells. Early descriptions of these cells labeled them as "glue" with a primarily passive structural/supportive role. However, with the advent of patch clamping and fluorescent calcium dye techniques from the late 1980s through the early 2000s, researchers have found that the role of these cells is much more extensive and complex (Araque et al. 2001). Glia are essential for neuronal development and survival, as well as for regulating synaptic function, brain metabolism, and cerebral blood flow. These roles are evolutionarily conserved across different phyla, demonstrating the importance of glial cells in regulating neuronal function and pathology in the CNS.

\section{Role of Glia in Manganese Neurotoxicity}

Manganese (Mn) neurotoxicity, or manganism, is a neurodegenerative disease of the cerebral cortex and basal ganglia caused by excessive exposure to $\mathrm{Mn}$ and is characterized by motor deficits that resemble those seen in idiopathic Parkinson's disease (PD), such as gait disturbances, facial masking, hypoxia and dysphonia, dystonia and action, and postural tremor (Guilarte 2010; Perl and Olanow 2007). However, there are clear neurological distinctions from PD, including a typical lack of resting tremor, distinct gait abnormalities, and differential involvement of neurons in the substantia nigra pars compacta. These PD-like manifestations are due to the neuropathological changes including neuronal loss, atrophy and gliosis within the globus pallidus (GP), substantia nigra pars reticulata (SNpr), and striatum (ST) of exposed individuals (Aschner and Aschner 1991; Sigel 2007). Typically, exposures to high levels of Mn occur occupationally in welders, miners, and steel workers (Hua and Huang 1991); however, the neurological consequences of environmental exposure to low levels of $\mathrm{Mn}$ through ingestion of crops with residues of the Mn-containing pesticide Maneb (Santamaria 2008) and well water with high concentrations of Mn (Woolf et al. 2002) are under scrutiny as an important route for nonoccupational exposure to the general population. In particular, there is increased concern with chronic Mn exposure in children due to their lower ability to clear Mn (Collipp et al. 1983); higher levels of iron deficiency, which have shown to elevate brain Mn levels (Aschner and Aschner 2005); and greater absorption of Mn from the GI tract (Neal and Guilarte 2012). Recent epidemiological studies have reported cognitive deficits in children exposed to high levels of Mn in drinking water (Menezes-Filho et al. 2011; Riojas-Rodriguez et al. 2010; Kim et al. 2009), highlighting the need for future studies addressing the long-term consequences of these exposures.

The mechanisms of how Mn exposure leads to specific neurodegenerative changes in the basal ganglia of exposed humans and animals are poorly understood. Elevated levels of $\mathrm{Mn}$ are routinely documented in the basal ganglia of exposed humans and animals (Olanow 2004), and experimental evidence has shown that Mn can be directly neurotoxic through 
inhibition of mitochondrial respiration leading to energy failure and oxidative stress (Zhang et al. 2003) and through excitotoxicity (Centonze et al. 2001). Other established mechanisms such as oxidative stress, glial toxicity, and neuroinflammation are also implicated in the progression of Mn neurotoxicity. Notably, the disorder will continue to progress both clinically and in rodent models of the disease even after cessation of exposure, suggesting ongoing mechanisms linked to progression that may include both unfolded protein stress and neuroinflammation (Sigel 2007; Aschner and Aschner 2005; Filipov and Dodd 2012).

The involvement of glia in Mn-induced neurotoxicity has only received increased attention over the past 20 years as a fundamental mechanism in the progression of Manganism (Filipov and Dodd 2012). Although activated astrocytes and microglia were often noted in post mortem evaluation of Mn-exposed patients (Perl and Olanow 2007), few studies examined the functional consequences or mechanisms of glial activation following exposure to Mn. This was most likely due to the ability of Mn to be directly toxic to neurons through inhibition of mitochondrial respiration and induction of oxidative stress (Zhang et al. 2003) and the historical focus on acute, high-level exposures. A study in 1998 by Spranger et al. (1998) changed the perceptions of glia involvement in Manganism by reporting that exposure to low concentrations of Mn could amplify inflammatory activation of glial cells and enhance neurotoxicity. Other studies have now built upon these initial findings revealing that Mn can exacerbate the effects of LPS and cytokines on activation of both microglia and astrocytes that causes dramatic potentiation in production of TNFa, IL-1 $\beta$, ROS, and NOS2 expression (Barhoumi et al. 2004; Chen et al. 2006; Filipov et al. 2005; Moreno et al. 2008, 2011). Increased levels of these and other inflammatory genes have also been measured in both rodent (Moreno et al. 2009; Zhao et al. 2009) and nonhuman primate (Verina et al. 2011) studies with deletion or inhibition of these pathways showing neuroprotection (Zhao et al. 2009; Streifel et al. 2012; Zhang et al. 2009).

\section{Neuroinflammation in Manganese Toxicity}

\section{Overview of Neuroinflammation in the CNS}

It is now clear that Mn exposure, even early in life, can have lasting effects on the neuroinflammatory status of glial cells (Moreno et al. 2009). Thus, neuroinflammatory activation of glia may be a fundamental mechanism in determining long-term neurological outcomes from Mn exposure. Astrocytes and microglia serve a multitude of essential functions within the CNS including integral roles in the innate immune system of the brain (Wyss-Coray and Mucke 2002). In response to foreign or endogenous signals, both astrocytes and microglia adopt an activated phenotype resulting in the release of proinflammatory mediators (Craft et al. 2005). This inflammatory system, known as neuroinflammation, is essential in normal tissue repair and in defense against foreign invasion; however, when sustained, this process can become deleterious through the release of neurotoxic factors that amplify underlying disease (Mosley et al. 2006; Glass et al. 2010; Tansey et al. 2007).

In normal circumstances, the neuroinflammatory reaction has auto regulatory mechanisms in place to limit the extent of activation as the process is neither discriminatory or specific (Wyss-Coray and Mucke 2002; Glass et al. 2010). For sustained inflammation to occur, there 
must be failure of self-resolution mechanisms or the presence of endogenous or environmental factors that are perceived as a threat. There are a variety of factors known to elicit activation of both microglia and astrocytes including products released by injured neurons such as glutamate (Kaushal and Schlichter 2008), ATP (Di Virgilio et al. 2009), and matrix metalloproteinase-3 (Kim et al. 2005); cytokines including interferon gamma (IFN $\gamma$ ), interleukin-1 $\beta$ (IL-1 $\beta$ ), and interleukin-6 (IL-6); adhesion molecules; growth factors; bloodderived factors; ionic imbalances; activation of complement products from viruses and bacteria; and presence of reactive oxygen species (Wyss-Coray and Mucke 2002; Sofroniew and Vinters 2010; Gehrmann et al. 1995). Furthermore, new evidence suggests that both microglia and astrocytes express endogenous pattern recognition receptors (PRRs) that respond to a variety of damage-associated molecular patterns (DAMPs) that results in molecular signaling events that promote inflammation and disease progression (Glass et al. 2010). These PRRs become activated in response to signals released by necrotic neurons or other pathologic products produced during disease including oxidized proteins and lipids (Husemann et al. 2002), messenger ribonucleic acid (mRNA), fibronectin, hyaluronic acid, heat shock proteins, amyloid-beta, neuromelanin, and alpha-synuclein (Block and Hong 2005; Gensel et al. 2012; Zhang et al. 2005). The production of inflammatory mediators is further increased by activated glia, leading to a feed-forward cycle of inflammation and further release of neurotoxic mediators of tissue injury.

Activated glia release diverse inflammatory factors including cytokines, chemokines, reactive oxygen species (ROS), and nitric oxide (NO) that are toxic to neurons (Kim et al. 2005; Gonzalez-Scarano and Baltuch 1999). Cytokines such as tumor necrosis factor-alpha (TNFa) and interleukin-6 (IL-6) are often upregulated very quickly in activated glial cells and can directly amplify inflammation through recruitment of both innate and adaptive immune cells, leading to neuronal apoptosis (Gensel et al. 2012; Gonzalez-Scarano and Baltuch 1999). Reactive oxygen species from Mn exposure can also damage neurons directly by increasing lipid peroxidation and mitochondrial dysfunction, causing subsequent energy failure, protein modifications, and DNA damage (Mosley et al. 2006). The formation of peroxynitrite, a by-product of superoxide and NO, is thought to be a major contributor to neuronal-induced cell death through nitration and nitrosylation of tyrosine and serine residues of proteins leading to impairment of normal cellular functions (McCarty 2006). Mn exposure results in significant increases in protein nitrosylation, indicative of nitrosative stress from NO production by glial cells (Moreno et al. 2009). Inhibition or deletion of many of these pathways has shown to be neuroprotective, but often the neuroprotection achieved is dependent on the timing of inhibition as often early downregulation of inflammation has actually worsened neuronal injury (Frank-Cannon et al. 2009). However, mice lacking the inducible form of NO synthase (iNOS/NOS2) are protected from Mn neurotoxicity, demonstrating the importance of this glial inflammatory pathway in the mechanism of neuronal injury (Streifel et al. 2012). Due to the complicated nature of neuroinflammation and the vast majority of implicated factors, systematic and thorough understanding is vital to understanding the implications that may come from targeting this pathway. 


\section{Glial Cell Activation in Neuroinflammation}

The activation of microglia and astrocytes is one of the universal components of neuroinflammation and is implicated in the progression of neurodegeneration in ischemia, seizure, Alzheimer's disease, multiple sclerosis, amyotrophic lateral sclerosis, Parkinson's disease (PD), and manganism (Mosley et al. 2006; Glass et al. 2010; Block and Hong 2005; Hirsch and Hunot 2009; Vezzani et al. 2013). Since the first early descriptions of activated glia in neurodegenerative diseases, there have been an increasing number of CNS pathologies described as having an association with activated glia. Although the regional pattern of neuroinflammation can vary among different disorders, there are common mechanisms by which activated glial cells sense stress and injury within the CNS and consequently transduce signals that amplify inflammatory activity of surrounding microglia and astrocytes (Glass et al. 2010). Research aimed at elucidating the pathogenesis of neuroinflammation is quickly expanding to understand the importance of this mechanism in the progression of many neuropathologies, including manganism. In this regard, it is useful to compare the role of neuroinflammation in Mn neurotoxicity to that of other better studied disorders, such as Parkinson's disease, to develop an appreciation for the mechanisms that are common to degenerative conditions of the CNS. The molecular regulation of neuroinflammatory gene expression in glial cells shares important commonalities between astrocytes, microglia, and peripheral immune cells such as monocytes.

Glial inflammatory activation is regulated by several different pathways including mitogenactivated protein kinases (MAPKs), activator protein-1 (AP-1), Janus kinase (JAK)/signal transducer and activator of transcription (STAT), and interferon regulator factor families (Glass et al. 2010); nevertheless, the nuclear factor kappa B (NF- $\mathrm{kB}$ ) appears to be the primary pathway involved in the activation of pro-inflammatory genes (Karin 2005). Deletion of NF- $\kappa B$ is detrimental to the ability of the immune system to initiate immunoprotective responses. Mice deficient in this pathway often succumb to opportunistic infections (Alcamo et al. 2001). Genetic deletion of this pathway in specific glial cells within the CNS has shown to be very neuroprotective with better recovery after spinal cord injury (Brambilla et al. 2005), decreased pathology in mouse models of multiple sclerosis (van Loo et al. 2006), and decreased seizure-induced neuronal death in kainic acid model of seizure (Cho et al. 2008).

NF- $\kappa B$ represents a family of transcription factors that are regulated by inhibitory $\mathrm{\kappa BS}$ (IKBS). Upon signal activation, IкBS are phosphorylated by IкB kinase complex (IKK) marking them for polyubiquitination and, ultimately, degradation by the 26 s proteasome, thus freeing the transcription factors, located as dimers within the cytosol, to translocate into the nucleus (DiDonato et al. 1997). The IKK complex consists of three different proteins including the two catalytic units IKKa/IKK1 and IKK $\beta / \mathrm{IKK} 2$ and the regulatory subunit IKK $\gamma$. These two catalytic subunits mark the division of the two NF- $\kappa B$ activation pathways known as the classical pathway and the alternative pathway. The classical NF- $\kappa B$ pathway involves the heterodimers of p50 and p65/RelA and is activated by the action of IKK $\beta /$ IKK2 of the IKK complex. This pathway is primarily involved in immunoregulation controlling innate immune responses and survival of immune cells. The alternative pathway is primarily involved in the development of secondary lymphoid organs and requires only IKKa/IKK1 
and results in the processing of p100 (Karin 1999; Li et al. 2003; Bonizzi and Karin 2004). Deletion of IKK $\beta / \mathrm{IKK} 2$ and not IKKa/IKK1 recapitulates similar mouse phenotypes as RelA knockout mice with almost complete inhibition of inflammatory responses and thus represents a major target in modulating glia neuroinflammatory activation (Alcamo et al. 2001). As detailed below, the NF- $\times B$ pathway is an important target of Mn in glial cells that integrates multiple extra- and intracellular stress signals to activate inflammatory gene expression.

\section{Manganese and Astrocytes}

\section{Description and Distribution of Astrocytes}

Astrocytes accumulate higher levels of Mn than neurons and are therefore considered an important target cell for transport of $\mathrm{Mn}$ into the brain as well as for initiating inflammatory signaling during neuronal stress and injury. Astrocytes encompasses a heterogeneous population of cells that can have vastly different morphological and physiological characteristics depending on their location with the brain (Matyash and Kettenmann 2010). Their morphological forms range from the protoplasmic astrocyte with extensive arborization found in the gray matter to the more rodlike fibrous astrocyte located within the white matter (Sofroniew and Vinters 2010; Perea and Araque 2010). With their extensive processes, they make contacts with neuronal bodies, synapses, axons, blood vessels, and other astrocytes, thereby creating a vast network that allows them to serve a multitude of both structural and important physiological roles within the CNS.

Astrocytes are the most numerous type of cell of the CNS, making up 60-70\% of all cells in the brain and also comprise $90 \%$ of all glial cells. Astrocytes are found throughout the CNS in a contingent but nonoverlapping manner that comprises distinct microdomains which enable them to make contact with a large number of neurons and with the microvasculature (Sofroniew and Vinters 2010). Astrocytes are morphologically characterized by expression of the intermediate filament proteins glial fibrillary acidic protein (GFAP) and vimentin. Other known markers of astrocytes in the adult brain include glutamine synthetase (GS), S100 calcium-binding protein- $\beta$, and glutamate transporters GLT-1/EAAT2 and GLAST/ EAAT1 (Kimelberg 2004); however, GFAP has been shown to be the most consistent marker in both physiological and pathological states (O'Callaghan and Sriram 2005).

\section{Functional Roles of Astrocytes Relevant to Manganese Neurotoxicity}

The first noted function of astrocytes within the adult CNS was purely structural; astrocytes were described as a scaffold to arrange and contain the neuronal circuitry due to their relative abundance and formation of glial scars in disease. Although it is now known that astrocytes have more complex roles, their formation of a continuous syncytium is still important for the structural integrity of the brain. These vast networks help to create specific micro and macro domains and help to create physical barriers between neuronal synapses (Sofroniew and Vinters 2010). Furthermore, astrocytic end feet are an important component of the glia limitans, a barrier that helps to isolate the brain parenchyma from the vasculature and subarachnoid compartments (Nimmerjahn 2009), as well as the blood-brain barrier 
(BBB) through the ensheathing of blood vessels throughout the CNS (Carmignoto and Gomez-Gonzalo 2010).

Past their structural roles, astrocytes serve as important facilitators of neuronal homeostasis through nutritive and trophic support. As a primary component of the BBB, astrocytes that surround endothelial cells are enriched in glucose receptors and channels and act as the main vehicle for the movement of glucose and oxygen from the blood to neurons. Astrocytes, but not neurons, are capable of storing glucose in the form of glycogen and of de novo synthesis of glutamate, which forms the basis for the functional metabolic coupling between these two cell types that maintains neuronal homeostasis (Parpura et al. 2012). Glutamate is the primary excitatory neurotransmitter in the brain, and its synaptic concentration is tightly regulated by astrocytes, which rapidly removed glutamate from synapses, where it can be safely transaminated to glutamine for recycling to neurons in the glutamate-glutamine cycle. Eighty percent of glutamate released into the synapse is removed by astrocytes and then converted to glutamine by GS, thereby preventing excitotoxic injury to neurons. This glutamine is released and then taken up by neurons that convert glutamine into glutamate and $\gamma$-amino butyric acid (GABA). Additionally, production of lactate by astrocytes is used by neurons to produce pyruvate and generate adenosine triphosphate (ATP) via the tricarboxylic acid cycle (TCA). These metabolically coupled support pathways in astrocytes are critical for neuronal survival and are important targets of Mn during neurotoxic exposures. Notably, Mn exposure results in marked increases in excitatory neurontransmission that likely damages neurons, supported by studies demonstrating the efficacy of the ionotropic glutamate receptor antagonist, MK-801, in preventing neuronal injury from chronic exposure to $\mathrm{Mn}$ (Xu et al. 2010).

In addition to being critical for neuronal metabolism, astrocytes are required for normal synaptic transmission through regulation of neurotransmitters, ions, water, and extracellular pH (Sofroniew and Vinters 2010). Astrocytes surround both pre- and postsynaptic terminals to form what is known as the tripartite synapse, allowing astrocytes to not only regulate neurotransmitters but also actively respond to and modulate synaptic plasticity through the release of gliotransmitters (Araque et al. 2001; Perea and Araque 2010; Nedergaard and Verkhratsky 2012; Perea et al. 2009). Astrocytes express a wide assortment of functional neurotransmitters including glutamate, GABA, dopamine, adrenalin/epinephrine, histamine, and glycine, the expression of which varies depending on the local microenvironment to match the physiology of their neuronal neighbors (Parpura et al. 2012). The majority of the neurotransmitter receptors expressed are metabotropic receptors coupled to G-proteins whose activation results in the generation of inositol triphosphate (IP3) and the release of calcium $\left(\mathrm{Ca}^{2+}\right)$. Astrocytes express at least three types of ionotropic receptors: $a$-amino-3hydroxy-5-methyl-isoxazole propionate (AMPA), N-methyl-D-aspartate (NMDA) types of tetrameric glutamate receptors, and P2X trimeric purinoreceptors (Lalo et al. 2008).

Activation of glia metabotropic and inotropic receptors results in the generation of $\mathrm{Ca}^{2+}$ waves within astrocytes that are propagated between astroglial networks through connexin gap junctions and glia release of ATP and glutamate (Araque et al. 2001; Kim and de Vellis 2005). This intercommunication between astrocytes is dynamic and is influenced by the extent of and frequency of neurotransmitter release which is important in the modulation of synapses in both learning and memory (Perea et al. 2009). It was recently reported that Mn 
disrupts ATP-dependent $\mathrm{Ca}^{2+}$ signaling in astrocytes by inhibiting entry of $\mathrm{Ca}^{2+}$ through the plasma membrane subsequent to activation of P2Y purinergic receptors (Streifel et al. 2013), suggesting that $\mathrm{Ca}^{2+}$-dependent homeostatic processes in astrocytes could be an important target of Mn that likely impacts neuronal physiology.

Calcium-based communication between astrocytes not only plays a large role in synaptic plasticity but is vital to the regulation of blood flow in response to neuronal activity known as neurovascular coupling (Sofroniew and Vinters 2010; Carmignoto and Gomez-Gonzalo 2010). In areas of high neuronal activity, elevations in calcium in astrocytes result in release of vasoactive compounds such as nitric oxide $(\mathrm{NO})$, prostaglandin $\mathrm{E} 2\left(\mathrm{PGE}_{2}\right)$, potassium $(\mathrm{K}$ ${ }^{+}$), and epoxygenase derivatives (EETs) at astrocytic end feet that results in a dilation or constriction of local vasculature (Nimmerjahn 2009; Mulligan and MacVicar 2004). This control of cerebral blood flow is complex, and the elucidations of how astrocytes cause specific vasodilation versus vasoconstriction in response to neuronal activity are still being fully elucidated. Mn can disrupt ATP-induced $\mathrm{Ca}^{2+}$ signaling and intercellular $\mathrm{Ca}^{2+}$ waves in astrocytes (Streifel et al. 2012), which could be detrimental to neuronal trophic support, rendering affected brain regions both focally hypoxic and with insufficient metabolic support. In this regard, even low levels of $\mathrm{Mn}^{2+}$ can disrupt ATP-dependent calcium signaling in astrocytes, in part through inhibition of TRPC 3 cation channels, which could alter the regulation of cerebral blood flow and therefore negatively impact neuronal homeostasis (Streifel et al. 2013). Inhibition of ATP-dependent calcium signaling in primary astrocytes has also been described for the cationic neurotoxicants, 1-methyl-4phenylpyridinium $\left(\mathrm{MPP}^{+}\right)$, and 6-hydroxydopamine, suggesting that disruption of homeostatic calcium signaling in astrocytes may be a common mechanism of injury for structurally diverse compounds affecting dopaminergic brain regions (Streifel et al. 2014).

Astrocytes are thus diverse and important regulators of neuronal metabolism and activity in the developed CNS; likewise, they also play an important role in the developing CNS, through neuronal guidance and synaptogenesis, and in adult neurogenesis (Doetsch 2003). In development, boundaries created by astrocytes help the migration of axons and neuroblasts, whereas the release of thrombospondin from astrocytes directs synapse formation. Furthermore, tagging of formed synapses with complement protein, C1q, helps tags synapses for pruning and removal (Christopherson et al. 2005; Powell and Geller 1999). In the adult CNS, neurogenesis within the subventricular zone of the olfactory bulb and the hippocampus is regulated by secretion of astrocytic factors such as Wnt3, interleukin- $1 \beta$ (IL-1 $\beta$ ), interleukin-6, and insulin-like growth factor-binding protein 6 (Parpura et al. 2012). Additionally, astrocytes themselves are believed to be the source of newly generated neurons determined by labeling based lineage tracking experiments (Doetsch 2003). Thus, neuronal generation, function, and continued survival are intimately linked and dependent on the vast and extensive physiology of their astrocytic counterparts.

\section{Role of Astrocytes in Neuroinflammation}

Activation of astrocytes is a biological reaction that is documented in most CNS diseases, as measured by increased expression of GFAP and alterations in astrocyte morphology that are considered early indicators of neuropathology (O'Callaghan and Sriram 2005; Parpura et al. 
2012). Activation of astrocytes can be neuroprotective through isolation of damage, glutathione production, BBB repair, and release of neurotrophic factors such as neural growth factor and glial-derived growth factor (Sofroniew and Vinters 2010; Block and Hong 2005; Kuno et al. 2006); however, astrogliosis can also be neurotoxic and promote disease progression. Detrimental consequences of astrogliosis include inhibition of axonal regeneration (Block and Hong 2005; Silver and Miller 2004), exacerbation of inflammation via cytokine production (Brambilla et al. 2005, 2009), production of reactive oxygen and nitrogen species (Hamby et al. 2006; Liu et al. 2006; Carbone et al. 2009), and excessive release of glutamate (Takano et al. 2009). Additionally, chronic inflammatory stimulation of astrocytes reduces glial capacity to generate and release neurotrophic mediators and execute normal physiological functions (Parpura et al. 2012). We and others have reported extensively on reactive astrocytosis in rodent models of manganism (Moreno et al. 2009, 2011; Streifel et al. 2012; Liu et al. 2006).

The regulation of astrocyte activation is under the control of many factors including cytokines IL-6, IFN $\gamma$, tumor necrosis factor-alpha (TNFa), toll-like receptor activators, neurotransmitters, ATP, reactive oxygen species, hypoxia, glucose deprivation, ammonia, and protein aggregates (Sofroniew and Vinters 2010; Parpura et al. 2012). Frequently, these activators are by-products of already injured neurons or factors released by activated microglia which indicate that astrocyte activation is often later in disease progression (Hirsch and Hunot 2009). Recent studies suggest that a-synuclein may be protective against Mn neurotoxicity, implicating protein misfolding in neurons as an important pathogenic mechanism following exposure to Mn (Harischandra et al. 2015). However, astrogliosis is often more persistent than microgliosis and is believed to be important in amplifying inflammatory processes and thereby inducing greater damage (Saijo et al. 2009). Moreover, in vitro studies have shown that isolated human astrocytes and not microglia are the major source of NO-induced neurotoxicity indicating they may be more significant in neuroinflammatory-induced neuronal death in humans than have been indicated in rodent models (Lee et al. 1993).

\section{Neuroinflammatory Effects of Manganese in Astrocytes}

Neuropathology in manganism is associated with robust astrogliosis in the basal ganglia, particularly the globus pallidus, subthalamic nucleus, and substantia nigra pars reticulata (Olanow 2004). Mn preferentially accumulates in astrocytes due to their expression of highcapacity transporters (Sidoryk-Wegrzynowicz and Aschner 2013), and therefore concentrations of $\mathrm{Mn}$ in astrocytes can be 50-60 times higher than in neurons, with the highest concentration of $\mathrm{Mn}$ found in the mitochondria (Sidoryk-Wegrzynowicz and Aschner 2013; Aschner et al. 1992; Morello et al. 2008). Similar to microglia, astrocytes release inflammatory cytokines and nitric oxide (NO) that influence the progression of neuronal injury during exposure to Mn (Moreno et al. 2008, 2009; Liu et al. 2006). In vitro studies demonstrate that human astrocytes are the primary source of NO-induced neurotoxicity, more so than microglia, suggesting that astrocytes could play a greater role in neuroinflammation-induced neuronal death from Mn than was initially demonstrated in rodent studies (Lee et al. 1993). Another in vitro study demonstrated that Mn inhibits the capacity of astrocytes to promote neuronal differentiation through a mechanism that involves 
oxidative stress and a reduction in levels of the extracellular matrix protein, fibronectin (Giordano et al. 2009). Oxidative stress in astrocytes also leads to dysfunction in mitochondria and, not surprisingly, a decreased production of ATP that could negatively impact neuronal function and survival (Barhoumi et al. 2004; Chen et al. 2006; Streifel et al. 2012).

Mn causes metabolic changes in astrocytic glucose metabolism by inhibition of the astrocyte-specific enzyme, glutamine synthetase (GS), thereby contributing to downregulation of glutamate transporters and compromising glutamate uptake (SuarezFernandez et al. 1999; Verkhratski and Butt 2013; Yin et al. 2007; Verkhratsky et al. 2016). These oxidative changes are consistent with the combined effects of Mn and inflammatory stimuli on mitochondrial function in astrocytes, which promotes greater production of ROS and deprecations in metabolic support of neurons, in addition to the damaging effects of inflammatory gene expression (Barhoumi et al. 2004). Such an effect would predispose neurons to excitotoxic injury due to the presence of excess synaptic glutamate resulting from dysfunction or downregulation of high-affinity astrocytic transporters. Additionally, $\mathrm{Mn}$ perturbs ATP-induced $\mathrm{Ca}^{2+}$ signaling and intercellular $\mathrm{Ca}^{2+}$ waves in astrocytes, which could negatively impact the ability of astrocytes to stimulate $\mathrm{Ca}^{2+}$-dependent increases in local cerebral blood flow in response to synaptic activity (Streifel et al. 2012; Tjalkens et al. 2006). Any failure of the capacity of astrocytes to supply adequate neurotrophic support during chronic overexposure to Mn could therefore lead to loss of trophic support, as well as excitotoxicity and neuronal death.

Mn exposure can also directly stimulate inflammatory gene expression in astrocytes through activation of NF- $\kappa \mathrm{B}$. This results in expression of COX2, NOS2, and multiple inflammatory cytokines and chemokines, leading to enhanced neuronal apoptosis (Araque et al. 2001; Moreno et al. 2008; Streifel et al. 2012; Carbone et al. 2009). Previous research from our laboratory demonstrated that gene deletion of Nos 2 in astrocytes protected against $\mathrm{Mn}$ induced neurological dysfunction in vivo and prevented NF- $\mathrm{kB}$-dependent neuronal injury from activated astrocytes exposed to $\mathrm{Mn}$ in vitro (Streifel et al. 2012). Neuroprotection in these studies was associated with decreased production of NO and inflammatory cytokines in Mn-treated astrocytes, highlighting the importance of inflammatory activation of these cells in the progression of neuronal injury from Mn exposure. These data also demonstrate that reactive inflammatory mediators such as NO are important contributors to Mn-induced neuronal dysfunction during exposure to $\mathrm{Mn}$.

\section{Manganese and Microglia}

\section{Description and Distribution of Microglia}

Microglia are the primary immunological effector cells of the brain, entering the CNS during embryonic development from a monocyte-derived cell type (Kim et al. 2005). As discussed below, microglia represent an important effector cell type during Mn neurotoxicity that respond rapidly with increased production of neuroinflammatory mediators. In the adult brain, microglia have very low rates of division, but their numbers can be replenished by perivascular mononuclear phagocytes (Gehrmann et al. 1995). They are heterogeneous through the adult brain and constitute 10 to $15 \%$ of all glial cells with greater numbers 
located within the gray matter. In particular, the highest concentrations of microglia are found within the olfactory bulb, hippocampus, and basal ganglia, including the substantia nigra, which holds the greatest density of microglia: $12 \%$ of all cells. Microglia exist in three different morphological states: a ramified phenotype found proximal to the neuropil, a rodlike state in fiber tracts, and a macrophage-like amoeboid shape in areas with an incomplete BBB (Lawson et al. 1990). Microglia are never at rest and are constantly migrating, but these migration patterns are distinct between different cells and do not overlap (Gehrmann et al. 1995).

\section{Functional Roles of Microglia Relevant to Manganese Neurotoxicity}

As with other macrophage-like cells resident in tissues, one of the primary functions of microglia in the CNS is immunosurveillance, and they possess dendritic and phagocytic functions similar to other monocyte-derived cells (Kim et al. 2005; Block and Hong 2005; Gonzalez-Scarano and Baltuch 1999). Microglia constantly move and sample the extracellular environment within their individual domains, clearing up debris via their phagocytic function as they migrate. They express a variety of neurotransmitter receptors, pattern recognition receptors (PRRs) and ionotropic receptors such as P2X7 to sense alterations in brain homeostasis, the presence of foreign materials and neuronal damage (Ransohoff and Perry 2009). Microglia represent the main class of cell involved in antigen presentation and are important in recruitment of immune cells such at $\mathrm{T}$ and $\mathrm{B}$ lymphocytes to sites of injury (Gehrmann et al. 1995; Gonzalez-Scarano and Baltuch 1999). Notably, microglia express the NOD-like receptor, NLRP3, which is critical to increasing expression of IL- $1 \beta$ via inflammasome activation and which can be potently activated by high levels of extracellular ATP through P2X7 receptors (Surprenant and North 2009). Astrocytes can release high concentrations of ATP in response to neuronal stress and injury, which suppresses neuronal excitability but can also stimulate activation of microglia. Mn may therefore stimulate neuroinflammatory activation of microglia both directly, as well as indirectly through its effects on astrocytes, suggesting several pathways by which glial-glial interactions may stimulate neuroinflammatory injury during Mn exposure.

More recently, research has determined that microglia may also play integral roles in neuronal development and migration. Amoeboid microglia are implicated in synaptic remodeling and regulation of neuronal apoptosis through the release of soluble factors and phagocytic pruning of synapses in late embryonic development (Block and Hong 2005). Furthermore, studies have shown microglia to release growth and neurotrophic factors during synaptogenesis (Nakajima and Kohsaka 1993). However, the role of microglia in mediating both trophic and neurotoxic cell-cell interactions during Mn neurotoxicity is still not well known.

\section{Role of Microglia in Neuroinflammation}

Microglia are the primary effectors of the innate immune response within the CNS, with activation occurring early in states of disease and often preceding overt neuropathology (Gehrmann et al. 1995; Hirsch and Hunot 2009). Under physiological conditions, microglia exist in a resting, ramified state releasing both anti-inflammatory and neurotrophic factors while surveying their domains (Streit 2002). However, in the presence of viral or bacterial 
products (Glass et al. 2010), ATP, changes in ion or neurotransmitter homeostasis (Mastroeni et al. 2009), cytokines such as IFN $\gamma$ and interleukin-4 (Gehrmann et al. 1995), colonystimulating factors (CSFs) (Kim and de Vellis 2005), and a list of other pathological products, microglia transform into an activated phenotype, proliferate, and migrate to the site of injury (Block and Hong 2005). Activation occurs in two stages. In the first stage, microglia adopt a rodlike shape and increase expression of major histocompatibility complex II (MHCII) and other inflammatory molecules. In the second stage, microglia morph into an amoeboid cell capable of phagocytosis (Gehrmann et al. 1995; Kim and de Vellis 2005).

Once activated, microglia can be both beneficial and deleterious in disease as they release both pro- and anti-inflammatory factors (Block and Hong 2005). Determining whether microglia neuroinflammatory responses will be helpful or toxic is often predicted by adoption of either the M1 known as "classical activation" or M2, the "alternative activation," phenotype (David and Kroner 2011).The M1 phenotype is primarily inflammatory with microglia upregulating MHCII, CD86, CD32, and CD16 with the production of TNFa, IL-1 $\beta$, and IL-6. In contrast, the M2 phenotype is more closely associated with tissue repair with increased expression of arginase 1 and CD206, as well as increased release of brainderived neurotrophic factor (BDNF), insulin-like growth factor-1 (IGF-1), and interleukin-10 (IL-10) (Kigerl et al. 2009).

The classical activated or M1 microglia are known to elicit neuronal death and perpetuate inflammation through release of a variety of cytotoxic substances such as NO, superoxide anion, cytokines, glutamate, prostaglandins, and aspartate (Takano et al. 2009; Mastroeni et al. 2009). They appear to be the major initial sensors of foreign or endogenous signals, secreting inflammatory mediators such as TNFa and IL- $1 \beta$ that can act on astrocytes to induce secondary inflammatory responses (Lee et al. 2012). Furthermore, prevention of microglial activation pharmacologically or genetically often protects against neuroinflammatory pathology, thus placing them as important regulators of inflammatory mechanism in neurodegenerative diseases (Block and Hong 2005; Cho et al. 2008).

\section{Neuroinflammatory Effects of Manganese in Microglia}

Resting or ramified microglia can release anti-inflammatory and neurotrophic factors. However, when activated microglia release neuroinflammatory mediators and proinflammatory cytokines such TNFa, IL-1 $\beta$, IL-6, and as well as reactive oxygen and nitrogen species (ROS and RNS), all of which can act on astrocytes to amplify inflammatory responses in the CNS (Liu et al. 2003, 2006; Lee et al. 2012; Chao et al. 1992). Rapid expression of these cytokines in microglia early in CNS injury suggests that such glial inflammatory responses are integral to neuronal injury from a variety of exogenous insults (Gensel et al. 2012; Gonzalez-Scarano and Baltuch 1999). Exposure to Mn causes activation of both microglia (Chang and Liu 1999) and astrocytes (Spranger et al. 1998), resulting in increased production of numerous inflammatory factors that cause neuronal injury (Filipov et al. 2005). Manganese can also enhance the inflammatory effects of other microglial activators, such as lipopolysaccharide (LPS), which amplifies neuronal injury in models of Mn neurotoxicity (Filipov and Dodd 2012; Filipov et al. 2005; Park and Chun 2016). 
However, the molecular mechanisms underlying these responses to $\mathrm{Mn}$ in microglia are still not completely understood. For example, Mn and LPS can synergistically enhance activation and inflammatory gene expression in microglia (Park and Chun 2016), consistent with transformation into a phagocytic phenotype that can lead to neuronal injury (Diaz-Aparicio et al. 2016). This may be directly relevant to Mn-induced neuronal injury in vivo, because blockade of phagocytosis may prevent some forms of inflammatory neurodegeneration (Neher et al. 2011).

Manganese also activates NF- $\kappa$ B in microglia and enhances the effects of LPS, resulting in inflammatory gene expression and production of inflammatory mediators leading to neuronal death (Filipov and Dodd 2012; Filipov et al. 2005; Park and Chun 2016). In BV-2 microglial cells treated with LPS, NF- $\kappa B$-dependent inflammatory gene expression by pharmacological modulators of the nuclear receptor, Nurr1, which stabilizes nuclear corepressor proteins and reduces binding of NF- $\mathrm{kB} / \mathrm{p} 65$ to inflammatory gene promoters (Saijo et al. 2009; De Miranda et al. 2015a). Thus, the inflammatory effects of Mn in microglia are tightly regulated both at the level of IKK activation as well as by nuclear proteins that modulate transcriptional activity of inflammatory genes. Evidence for the importance of NR4A1 (Nurr1) in regulating microglial activation was recently reported in studies using a novel pharmacological Nurr1 agonist that prevented glial activation and neuronal injury from the dopaminergic neurotoxicant, MPTP (1-methyl-4-phenyl-1,2,3,6tetrahydropyridine) (De Miranda et al. 2015b). This data suggest that targeting NR4A receptors in microglia could be a promising avenue for prevention of NF- $\kappa \mathrm{B}$ activation in glia and, thus, inflammatory neuronal injury from a variety of neurotoxicants, including $\mathrm{Mn}$.

Another research group recently reported that P2Y12 participates in ischemia-related inflammation by mediating microglial migration and potentiation of neurotoxicity (Webster et al. 2013). Upon inhibition or deficiency of the P2Y12 receptor in BV-2 microglia, there is less NF- $\kappa \mathrm{B}$ activation, suggesting an additional anti-inflammatory, neuroprotective benefit of the antiplatelet drug, clopidogrel. Mn potently affects glial signaling through P2Y and P2X receptors (Streifel et al. 2013, 2014), suggesting that mitigating these effects could potentially reduce NF- $\mathrm{kB}$-mediated neuroinflammation in glia following Mn exposure.

\section{Glial-Glial Cross Talk in Mn-Dependent Inflammatory Signaling}

Sustained inflammatory activation of microglia is implicated as an important mechanism in the progression of many neurodegenerative diseases including AD, PD, and manganism. Experimental models of PD (Hirsch and Hunot 2009) and manganism (Zhao et al. 2009) have often identified the transition of microglia from a resting to activated phenotype prior to overt neuropathology. Cell culture models show Mn potentiates microglia inflammatory gene expression in response to LPS/cytokine treatment through activation of pathways such as NF- $\mathrm{\kappa B}$ and mitogen-activated protein kinase (MAPK) (Filipov and Dodd 2012; Crittenden and Filipov 2011). Removal of microglia or use of antioxidants has shown to reduce neuronal loss indicating microglial activation may serve as a critical step in mediating neuronal injury during Mn exposure and that microglia also likely directly promote activation of astrocytes that then amplify neuronal damage (Zhao et al. 2009; Zhang et al. 2010). Evidence for this is provided by studies indicating that activated microglia can 
enhance the activation of adjacent astrocytes by releasing factors such TNF and IL- $1 \beta$ that can further magnify neuronal injury (Hirsch and Hunot 2009; Saijo et al. 2009). Microglial responses are often rapid, in contrast to the more delayed activation often seen in astrocytes, suggesting that temporally distinct signaling events are required for a reactive glial phenotype. Underscoring this point, decreased microgliosis in vivo is associated with reduced astrocytosis (Zhang et al. 2010). Despite the known role of microglial-astrocyte cross talk in $\mathrm{AD}$ and $\mathrm{PD}$, these important glial-glial interactions are less well understood in Mn neurotoxicity.

Astrocytes serve as the major homeostatic regulator and storage site for $\mathrm{Mn}$ in the brain (Araque et al. 2001; Aschner and Aschner 1991) and are a prominent contributor to Mnstimulated nitric oxide (NO) production through NOS2 (Zhang et al. 2009). We previously reported that $\mathrm{Mn}$ enhances the inductive effects of inflammatory cytokines on astrocyte expression of Nos 2 through stimulation of NF- $\mathrm{\kappa B}$ (Moreno et al. 2008) and astrocytes activated by exposure to $\mathrm{Mn}$ and inflammatory cytokine-induced apoptosis in co-cultured striatal neurons (Streifel et al. 2012). However, without co-treatment with cytokines, astrocytes are unable to cause neuronal apoptosis in response to Mn treatment (Spranger et al. 1998) indicating that microglia are likely required for initiation of neuroinflammatory mechanisms in astrocytes during Mn neurotoxicity.

Using immunopurified cultures of primary microglia and astrocytes, data from our laboratory demonstrate that microglia directly accumulate $\mathrm{Mn}$, which stimulates a mixed inflammatory phenotype characterized by release of IL-6, TNF, CCL2, and CCL5. These experiments also revealed that products from Mn-activated microglia are essential for neuroinflammatory activation of Mn-exposed astrocytes and that NF- $\mathrm{kB}$-dependent release of TNF from microglia is a key signaling event in microglia regulating these glial-glial interactions. To decipher the complex signaling mechanisms likely to influence development of a neuroinflammatory phenotype in Mn neurotoxicity, additional experimentation in primary astrocytes and microglia is required to determine the relative contributions of each cell type to a reactive phenotype following Mn exposure.

Despite the heightened focus on glial involvement in manganism, there are still many unanswered questions regarding mechanisms due to the limited number of in vivo studies and the inability of Mn to be a very potent glial activator in the absence of other inflammatory factors (Park and Chun 2016).As with other disorders of the CNS with a neuroinflammatory component, most studies into glial involvement in manganism have used single or mixed cultures of microglia or astrocytes, with few studies examining cell-cell interactions. The studies described in this review suggest that complicated signaling mechanisms between microglia and astrocytes likely underlie development of a neuroinflammatory phenotype Mn neurotoxicity that ultimately results in the progression of neuronal injury leading to psychological and motor manifestations of manganism. Microglia produce a large number of pro-inflammatory factors that could amplify the activation state of astrocytes, including TNFa and IL-1 $\beta$, as well as numerous cytokines and chemokines. It will be important in future studies to determine which of these factors are most relevant to the cell-cell interactions underlying the damaging effects of neuroinflammation following exposure to Mn. With limited or no treatment options for interdicting neuroinflammation in 
the CNS, it will be imperative to better identify underlying mechanisms in order to develop better therapies. This is particularly of concern, given the recent appreciation for how $\mathrm{Mn}$ exposures in children can lead to persistent adverse neurological affects. Thus, there is a need for a more systematic and comprehensive look at glial involvement and the potential importance of this response in chronic exposures.

\section{Conclusion}

Neuroinflammatory activation of glial cells is an important mechanism in Mn neurotoxicity and in other degenerative conditions of the CNS. Studies in the last several decades have redefined the importance of astrocytes and microglia to neuronal development, homeostasis, and survival, transforming our understanding of the role of these cells from inert structural components to important components of brain physiology and pathology. More specifically, the importance of astrocytes and microglia to neuronal survival has received increased attention, as these two glial types are the most often altered during disease states and are now known to be fundamental components of the innate immune system of the brain. Inflammatory activation of glia, or neuroinflammation, is a classic and conserved marker of neuropathology and is implicated in the progression, and possibly initiation, of several CNS disorders including seizure, Parkinson's disease, and manganism. Yet, much of the above information on neuroinflammation has been gleaned from rodent modeling with few studies utilizing translational or environmental relevant models to examine these important mechanisms. Furthermore, because glial activation can also serve either neuroprotective or neurotoxic functions, there exists a need to better understand the timeline and pathways of glial activation with a more extensive focus on the relative contributions of different glial types and the dynamics of glial-to-glial signaling. By examining specific glial-derived mechanisms in several neurodegenerative diseases, we may better understand the implications of neuroinflammation for CNS pathology and discover new potential targets for therapeutic intervention.

\section{References}

Alcamo E, et al. Targeted mutation of TNF receptor I rescues the RelA-deficient mouse and reveals a critical role for NF-kappa B in leukocyte recruitment. J Immunol 2001;167:1592-600. [PubMed: 11466381]

Araque A, Carmignoto G, Haydon PG. Dynamic signaling between astrocytes and neurons. Annu Rev Physiol 2001;63:795-813. 10.1146/annurev.physiol.63.1.795. [PubMed: 11181976]

Aschner M, Aschner JL. Manganese neurotoxicity: cellular effects and blood-brain barrier transport. Neurosci Biobehav Rev 1991;15:333-40. [PubMed: 1956602]

Aschner JL, Aschner M. Nutritional aspects of manganese homeostasis. Mol Asp Med 2005;26:35362. 10.1016/j.mam.2005.07.003.

Aschner M, Gannon M, Kimelberg HK. Manganese uptake and efflux in cultured rat astrocytes. J Neurochem 1992;58:730-5. [PubMed: 1729413]

Barhoumi R, Faske J, Liu X, Tjalkens RB. Manganese potentiates lipopolysaccharide-induced expression of NOS2 in C6 glioma cells through mitochondrial-dependent activation of nuclear factor kappaB. Brain Res Mol Brain Res 2004;122:167-79. 10.1016/j.molbrainres.2003.12.009. [PubMed: 15010209]

Block ML, Hong JS. Microglia and inflammation-mediated neurodegeneration: multiple triggers with a common mechanism. Prog Neurobiol 2005;76:77-98. 10.1016/j.pneurobio.2005.06.004.

[PubMed: 16081203] 
Bonizzi G, Karin M. The two NF-kappaB activation pathways and their role in innate and adaptive immunity. Trends Immunol 2004;25:280-8. 10.1016/j.it.2004.03.008. [PubMed: 15145317]

Brambilla R, et al. Inhibition of astroglial nuclear factor kappaB reduces inflammation and improves functional recovery after spinal cord injury. J Exp Med 2005;202:145-56. 10.1084/jem.20041918. [PubMed: 15998793]

Brambilla R, et al. Transgenic inhibition of astroglial NF-kappa B improves functional outcome in experimental autoimmune encephalomyelitis by suppressing chronic central nervous system inflammation. J Immunol 2009;182:2628-40. 10.4049/jimmunol.0802954. [PubMed: 19234157]

Carbone DL, Popichak KA, Moreno JA, Safe S, Tjalkens RB. Suppression of 1-methyl-4phenyl-1,2,3,6-tetrahydropyridine-induced nitric-oxide synthase 2 expression in astrocytes by a novel diindolylmethane analog protects striatal neurons against apoptosis. Mol Pharmacol 2009;75:35-43. 10.1124/mol.108.050781. [PubMed: 18840677]

Carmignoto G, Gomez-Gonzalo M. The contribution of astrocyte signalling to neurovascular coupling. Brain Res Rev 2010;63:138-48. 10.1016/j.brainresrev.2009.11.007. [PubMed: 19948187]

Centonze D, Gubellini P, Bernardi G, Calabresi P. Impaired excitatory transmission in the striatum of rats chronically intoxicated with manganese. Exp Neurol 2001;172:469-76. 10.1006/exnr. 2001.7812. [PubMed: 11716571]

Chang JY, Liu LZ. Manganese potentiates nitric oxide production by microglia. Brain Res Mol Brain Res 1999;68:22-8. [PubMed: 10320780]

Chao CC, Hu S, Molitor TW, Shaskan EG, Peterson PK. Activated microglia mediate neuronal cell injury via a nitric oxide mechanism. J Immunol 1992;149:2736-41. [PubMed: 1383325]

Chen CJ, et al. Manganese modulates pro-inflammatory gene expression in activated glia. Neurochem Int 2006;49:62-71. 10.1016/j.neuint.2005.12.020. [PubMed: 16488514]

Cho IH, et al. Role of microglial IKKbeta in kainic acid-induced hippocampal neuronal cell death. Brain 2008;131:3019-33. 10.1093/brain/awn230. [PubMed: 18819987]

Christopherson KS, et al. Thrombospondins are astrocyte-secreted proteins that promote CNS synaptogenesis. Cell 2005;120:421-33. 10.1016/j.cell.2004.12.020. [PubMed: 15707899]

Collipp PJ, Chen SY, Maitinsky S. Manganese in infant formulas and learning disability. Ann Nutr Metab 1983;27:488-94. [PubMed: 6651226]

Couper J On the effects of black oxide of manganese when inhaled into the lungs. British Annals of Medicine, Pharmacy, Vital Statistics, and General Science 1837;1:41-2.

Craft JM, Watterson DM, Van Eldik LJ. Neuroinflammation: a potential therapeutic target. Expert Opin Ther Targets 2005;9:887-900. 10.1517/14728222.9.5.887. [PubMed: 16185146]

Crittenden PL, Filipov NM. Manganese modulation of MAPK pathways: effects on upstream mitogen activated protein kinase kinases and mitogen activated kinase phosphatase- 1 in microglial cells. $\mathrm{J}$ Appl Toxicol 2011;31:1-10. 10.1002/jat.1552. [PubMed: 20589745]

David S, Kroner A. Repertoire of microglial and macrophage responses after spinal cord injury. Nat Rev Neurosci 2011;12:388-99. 10.1038/nrn3053. [PubMed: 21673720]

De Miranda BR, et al. The Nurr1 activator 1,1-Bis(3'-Indolyl)-1-(p-Chlorophenyl)methane blocks inflammatory Gene expression in BV-2 microglial cells by inhibiting nuclear factor kappaB. Mol Pharmacol 2015a;87:1021-34. 10.1124/mol.114.095398. [PubMed: 25858541]

De Miranda BR, et al. Novel para-phenyl substituted diindolylmethanes protect against MPTP neurotoxicity and suppress glial activation in a mouse model of Parkinson's disease. Toxicol Sci 2015b;143:360-73. 10.1093/toxsci/kfu236. [PubMed: 25406165]

Di Virgilio F, Ceruti S, Bramanti P, Abbracchio MP. Purinergic signalling in inflammation of the central nervous system. Trends Neurosci 2009;32:79-87. 10.1016/j.tins.2008.11.003. [PubMed: 19135728]

Diaz-Aparicio I, Beccari S, Abiega O, Sierra A. Clearing the corpses: regulatory mechanisms, novel tools, and therapeutic potential of harnessing microglial phagocytosis in the diseased brain. Neural Regen Res 2016;11:1533-9. 10.4103/1673-5374.193220. [PubMed: 27904472]

DiDonato JA, Hayakawa M, Rothwarf DM, Zandi E, Karin M. A cytokine-responsive IkappaB kinase that activates the transcription factor NF-kappaB. Nature 1997;388:548-54. 10.1038/41493. [PubMed: 9252186] 
Doetsch F The glial identity of neural stem cells. Nat Neurosci 2003;6:1127-34. 10.1038/nn1144. [PubMed: 14583753]

Filipov NM, Dodd CA. Role of glial cells in manganese neurotoxicity. J Appl Toxicol 2012;32:310-7. 10.1002/jat.1762. [PubMed: 22120544]

Filipov NM, Seegal RF, Lawrence DA. Manganese potentiates in vitro production of proinflammatory cytokines and nitric oxide by microglia through a nuclear factor kappa B-dependent mechanism. Toxicol Sci 2005;84:139-48. 10.1093/toxsci/kfi055. [PubMed: 15601679]

Frank-Cannon TC, Alto LT, McAlpine FE, Tansey MG. Does neuroinflammation fan the flame in neurodegenerative diseases? Mol Neurodegener 2009;4:47 10.1186/1750-1326-4-47. [PubMed: 19917131]

Gehrmann J, Matsumoto Y, Kreutzberg GW. Microglia: intrinsic immuneffector cell of the brain. Brain Res Brain Res Rev 1995;20:269-87. [PubMed: 7550361]

Gensel JC, Kigerl KA, Mandrekar-Colucci SS, Gaudet AD, Popovich PG. Achieving CNS axon regeneration by manipulating convergent neuro-immune signaling. Cell Tissue Res 2012;349:20113. 10.1007/s00441-012-1425-5. [PubMed: 22592625]

Giordano G, Pizzurro D, VanDeMark K, Guizzetti M, Costa LG. Manganese inhibits the ability of astrocytes to promote neuronal differentiation. Toxicol Appl Pharmacol 2009;240:226-35. 10.1016/j.taap.2009.06.004. [PubMed: 19524604]

Glass CK, Saijo K, Winner B, Marchetto MC, Gage FH. Mechanisms underlying inflammation in neurodegeneration. Cell 2010;140:918-34. 10.1016/j.cell.2010.02.016. [PubMed: 20303880]

Gonzalez-Scarano F, Baltuch G. Microglia as mediators of inflammatory and degenerative diseases. Annu Rev Neurosci 1999;22:219-40. 10.1146/annurev.neuro.22.1.219. [PubMed: 10202538]

Guilarte TR. Manganese and Parkinson's disease: a critical review and new findings. Environ Health Perspect 2010;118:1071-80. 10.1289/ehp.0901748. [PubMed: 20403794]

Hamby ME, Hewett JA, Hewett SJ. TGF-beta1 potentiates astrocytic nitric oxide production by expanding the population of astrocytes that express NOS-2. Glia 2006;54:566-77. 10.1002/glia. 20411. [PubMed: 16921522]

Harischandra DS, Jin H, Anantharam V, Kanthasamy A, Kanthasamy AG. Alpha-Synuclein protects against manganese neurotoxic insult during the early stages of exposure in a dopaminergic cell model of Parkinson's disease. Toxicol Sci 2015;143:454-68. 10.1093/toxsci/kfu247. [PubMed: 25416158]

Hirsch EC, Hunot S. Neuroinflammation in Parkinson's disease: a target for neuroprotection? Lancet Neurol 2009;8:382-97. 10.1016/S1474-4422(09)70062-6. [PubMed: 19296921]

Hua MS, Huang CC. Chronic occupational exposure to manganese and neurobehavioral function. J Clin Exp Neuropsychol 1991;13:495-507. 10.1080/01688639108401066. [PubMed: 1918281]

Husemann J, Loike JD, Anankov R, Febbraio M, Silverstein SC. Scavenger receptors in neurobiology and neuropathology: their role on microglia and other cells of the nervous system. Glia 2002;40:195-205. 10.1002/glia.10148. [PubMed: 12379907]

Karin M How NF-kappaB is activated: the role of the IkappaB kinase (IKK) complex. Oncogene 1999;18:6867-74. 10.1038/sj.onc.1203219. [PubMed: 10602462]

Karin M Inflammation-activated protein kinases as targets for drug development. Proc Am Thorac Soc 2005;2:386-390.; 10.1513/pats.200504-034SR. [PubMed: 16267367]

Kaushal V, Schlichter LC. Mechanisms of microglia-mediated neurotoxicity in a new model of the stroke penumbra. J Neurosci 2008;28:2221-30. 10.1523/JNEUROSCI.5643-07.2008. [PubMed: 18305255]

Kigerl KA, et al. Identification of two distinct macrophage subsets with divergent effects causing either neurotoxicity or regeneration in the injured mouse spinal cord. J Neurosci 2009;29:13435-44. 10.1523/JNEUROSCI.3257-09.2009. [PubMed: 19864556]

Kim SU, de Vellis J. Microglia in health and disease. J Neurosci Res 2005;81:302-13. 10.1002/jnr. 20562. [PubMed: 15954124]

Kim YS, et al. Matrix metalloproteinase-3: a novel signaling proteinase from apoptotic neuronal cells that activates microglia. J Neurosci 2005;25:3701-11. 10.1523/JNEUROSCI.4346-04.2005. [PubMed: 15814801] 
Kim Y, et al. Co-exposure to environmental lead and manganese affects the intelligence of school-aged children. Neurotoxicology 2009;30:564-71. 10.1016/j.neuro.2009.03.012. [PubMed: 19635390]

Kimelberg HK. The problem of astrocyte identity. Neurochem Int 2004;45:191-202. 10.1016/j.neuint. 2003.08.015. [PubMed: 15145537]

Kuno R, et al. The role of TNF-alpha and its receptors in the production of NGF and GDNF by astrocytes. Brain Res 2006;1116:12-8. 10.1016/j.brainres.2006.07.120. [PubMed: 16956589]

Lalo U, et al. P2X1 and P2X5 subunits form the functional P2X receptor in mouse cortical astrocytes. J Neurosci 2008;28:5473-80. 10.1523/JNEUROSCI.1149-08.2008. [PubMed: 18495881]

Lawson LJ, Perry VH, Dri P, Gordon S. Heterogeneity in the distribution and morphology of microglia in the normal adult mouse brain. Neuroscience 1990;39:151-70. [PubMed: 2089275]

Lee SC, Liu W, Dickson DW, Brosnan CF, Berman JW. Cytokine production by human fetal microglia and astrocytes. Differential induction by lipopolysaccharide and IL-1 beta. J Immunol 1993;150:2659-67. [PubMed: 8454848]

Lee DJ, Hsu MS, Seldin MM, Arellano JL, Binder DK. Decreased expression of the glial water channel aquaporin-4 in the intrahippocampal kainic acid model of epileptogenesis. Exp Neurol 2012;235:246-55. 10.1016/j.expneurol.2012.02.002. [PubMed: 22361023]

Li ZW, Omori SA, Labuda T, Karin M, Rickert RC. IKK beta is required for peripheral B cell survival and proliferation. J Immunol 2003;170:4630-7. [PubMed: 12707341]

Liu B, Gao HM, Hong JS. Parkinson's disease and exposure to infectious agents and pesticides and the occurrence of brain injuries: role of neuroinflammation. Environ Health Perspect 2003;111:106573. [PubMed: 12826478]

Liu X, Sullivan KA, Madl JE, Legare M, Tjalkens RB. Manganese-induced neurotoxicity: the role of astroglial-derived nitric oxide in striatal interneuron degeneration. Toxicol Sci 2006;91:521-31. 10.1093/toxsci/kfj150. [PubMed: 16551646]

Mastroeni D, et al. Microglial responses to dopamine in a cell culture model of Parkinson's disease. Neurobiol Aging 2009;30:1805-17. 10.1016/j.neurobiolaging.2008.01.001. [PubMed: 18325635]

Matyash V, Kettenmann H. Heterogeneity in astrocyte morphology and physiology. Brain Res Rev 2010;63:2-10. 10.1016/j.brainresrev.2009.12.001. [PubMed: 20005253]

McCarty MF. Down-regulation of microglial activation may represent a practical strategy for combating neurodegenerative disorders. Med Hypotheses 2006;67:251-69. 10.1016/j.mehy. 2006.01.013. [PubMed: 16513287]

Menezes-Filho JA, Novaes Cde O, Moreira JC, Sarcinelli PN, Mergler D. Elevated manganese and cognitive performance in school-aged children and their mothers. Environ Res 2011;111:156-63. 10.1016/j.envres.2010.09.006. [PubMed: 20943219]

Morello M, et al. Sub-cellular localization of manganese in the basal ganglia of normal and manganese-treated rats an electron spectroscopy imaging and electron energy-loss spectroscopy study. Neurotoxicology 2008;29:60-72. 10.1016/j.neuro.2007.09.001. [PubMed: 17936361]

Moreno JA, Sullivan KA, Carbone DL, Hanneman WH, Tjalkens RB. Manganese potentiates nuclear factor-kappaB-dependent expression of nitric oxide synthase 2 in astrocytes by activating soluble guanylate cyclase and extracellular responsive kinase signaling pathways. J Neurosci Res 2008;86:2028-38. 10.1002/jnr.21640. [PubMed: 18335517]

Moreno JA, Streifel KM, Sullivan KA, Legare ME, Tjalkens RB. Developmental exposure to manganese increases adult susceptibility to inflammatory activation of glia and neuronal protein nitration. Toxicol Sci 2009;112:405-15. 10.1093/toxsci/kfp221. [PubMed: 19812365]

Moreno JA, Streifel KM, Sullivan KA, Hanneman WH, Tjalkens RB. Manganese-induced NF-kappaB activation and nitrosative stress is decreased by estrogen in juvenile mice. Toxicol Sci 2011;122:121-33. 10.1093/toxsci/kfr091. [PubMed: 21512103]

Mosley RL, et al. Neuroinflammation, Oxidative Stress and the Pathogenesis of Parkinson's Disease. Clin Neurosci Res 2006;6:261-81. 10.1016/j.cnr.2006.09.006. [PubMed: 18060039]

Mulligan SJ, MacVicar BA. Calcium transients in astrocyte endfeet cause cerebrovascular constrictions. Nature 2004;431:195-9. 10.1038/nature02827. [PubMed: 15356633]

Nakajima K, Kohsaka S. Functional roles of microglia in the brain. Neurosci Res 1993;17:187-203. [PubMed: 8233123] 
Neal AP, Guilarte TR. Mechanisms of heavy metal neurotoxicity: lead and manganese. Drug Metab toxicol 2012;5

Nedergaard M, Verkhratsky A. Artifact versus reality--how astrocytes contribute to synaptic events. Glia 2012;60:1013-23. 10.1002/glia.22288. [PubMed: 22228580]

Neher JJ, et al. Inhibition of microglial phagocytosis is sufficient to prevent inflammatory neuronal death. J Immunol 2011;186:4973-83. 10.4049/jimmunol.1003600. [PubMed: 21402900]

Nimmerjahn A Astrocytes going live: advances and challenges. J Physiol 2009;587:1639-47. 10.1113/ jphysiol.2008.167171. [PubMed: 19204050]

O'Callaghan JP, Sriram K. Glial fibrillary acidic protein and related glial proteins as biomarkers of neurotoxicity. Expert Opin Drug Saf 2005;4:433-42. 10.1517/14740338.4.3.433. [PubMed: 15934851]

Olanow CW. Manganese-induced parkinsonism and Parkinson's disease. Ann N Y Acad Sci 2004;1012:209-23. [PubMed: 15105268]

Park E, Chun HS. Melatonin attenuates manganese and lipopolysaccharide-induced inflammatory activation of BV2 microglia. Neurochem Res 2016; 10.1007/s11064-016-2122-7.

Parpura V, et al. Glial cells in (patho)physiology. J Neurochem 2012;121:4-27. 10.1111/j. 1471-4159.2012.07664.x. [PubMed: 22251135]

Perea G, Araque A. GLIA modulates synaptic transmission. Brain Res Rev 2010;63:93-102. 10.1016/ j.brainresrev.2009.10.005. [PubMed: 19896978]

Perea G, Navarrete M, Araque A. Tripartite synapses: astrocytes process and control synaptic information. Trends Neurosci 2009;32:421-31. 10.1016/j.tins.2009.05.001. [PubMed: 19615761]

Perl DP, Olanow CW. The neuropathology of manganese-induced parkinsonism. J Neuropathol Exp Neurol 2007;66:675-82. 10.1097/nen.0b013e31812503cf. [PubMed: 17882011]

Powell EM, Geller HM. Dissection of astrocyte-mediated cues in neuronal guidance and process extension. Glia 1999;26:73-83. [PubMed: 10088674]

Ransohoff RM, Perry VH. Microglial physiology: unique stimuli, specialized responses. Annu Rev Immunol 2009;27:119-45. 10.1146/annurev.immunol.021908.132528. [PubMed: 19302036]

Riojas-Rodriguez $\mathrm{H}$, et al. Intellectual function in Mexican children living in a mining area and environmentally exposed to manganese. Environ Health Perspect 2010;118:1465-70. [PubMed: 20936744]

Saijo K, et al. A Nurr1/CoREST pathway in microglia and astrocytes protects dopaminergic neurons from inflammation-induced death. Cell 2009;137:47-59. 10.1016/j.cell.2009.01.038. [PubMed: 19345186]

Santamaria AB. Manganese exposure, essentiality \& toxicity. Indian J Med Res 2008;128:484-500. [PubMed: 19106442]

Sidoryk-Wegrzynowicz M, Aschner M. Role of astrocytes in manganese mediated neurotoxicity. BMC Pharmacol Toxicol 2013;14:23 10.1186/2050-6511-14-23. [PubMed: 23594835]

Sigel AS, Sigel H; Sigel RKO Metal Ions in Life Sciences (Wiley, 2007).

Silver J, Miller JH. Regeneration beyond the glial scar. Nat Rev Neurosci 2004;5:146-56. 10.1038/ nrn1326. [PubMed: 14735117]

Sofroniew MV, Vinters HV. Astrocytes: biology and pathology. Acta Neuropathol 2010;119:7-35. 10.1007/s00401-009-0619-8. [PubMed: 20012068]

Spranger M, et al. Manganese augments nitric oxide synthesis in murine astrocytes: a new pathogenetic mechanism in manganism? Exp Neurol 1998;149:277-83. 10.1006/exnr.1997.6666. [PubMed: 9454637]

Streifel KM, Moreno JA, Hanneman WH, Legare ME, Tjalkens RB. Gene deletion of nos2 protects against manganese-induced neurological dysfunction in juvenile mice. Toxicol Sci 2012;126:18392. 10.1093/toxsci/kfr335. [PubMed: 22174044]

Streifel KM, Miller J, Mouneimne R, Tjalkens RB. Manganese inhibits ATP-induced calcium entry through the transient receptor potential channel TRPC3 in astrocytes. Neurotoxicology 2013;34:160-6. 10.1016/j.neuro.2012.10.014. [PubMed: 23131343] 
Streifel KM, et al. Dopaminergic neurotoxicants cause biphasic inhibition of purinergic calcium signaling in astrocytes. PLoS One 2014;9:e110996 10.1371/journal.pone.0110996. [PubMed: 25365260]

Streit WJ. Microglia as neuroprotective, immunocompetent cells of the CNS. Glia 2002;40:133-9. 10.1002/glia.10154. [PubMed: 12379901]

Suarez-Fernandez MB, et al. Aluminum-induced degeneration of astrocytes occurs via apoptosis and results in neuronal death. Brain Res 1999;835:125-36. [PubMed: 10415367]

Surprenant A, North RA. Signaling at purinergic P2X receptors. Annu Rev Physiol 2009;71:333-59. 10.1146/annurev.physiol.70.113006.100630. [PubMed: 18851707]

Takano T, Oberheim N, Cotrina ML, Nedergaard M. Astrocytes and ischemic injury. Stroke 2009;40:S8-12. 10.1161/STROKEAHA.108.533166. [PubMed: 19064795]

Tansey MG, McCoy MK, Frank-Cannon TC. Neuroinflammatory mechanisms in Parkinson's disease: potential environmental triggers, pathways, and targets for early therapeutic intervention. Exp Neurol 2007;208:1-25. 10.1016/j.expneurol.2007.07.004. [PubMed: 17720159]

Tjalkens RB, Zoran MJ, Mohl B, Barhoumi R. Manganese suppresses ATP-dependent intercellular calcium waves in astrocyte networks through alteration of mitochondrial and endoplasmic reticulum calcium dynamics. Brain Res 2006;1113:210-9. 10.1016/j.brainres.2006.07.053. [PubMed: 16934782]

van Loo G, et al. Inhibition of transcription factor NF-kappaB in the central nervous system ameliorates autoimmune encephalomyelitis in mice. Nat Immunol 2006;7:954-61. 10.1038/ ni1372. [PubMed: 16892069]

Verina T, Kiihl SF, Schneider JS, Guilarte TR. Manganese exposure induces microglia activation and dystrophy in the substantia nigra of non-human primates. Neurotoxicology 2011;32:215-26. 10.1016/j.neuro.2010.11.003. [PubMed: 21112353]

Verkhratski AN, Butt A. Glial physiology and pathophysiology Chichester: Wiley-Blackwell; 2013.

Verkhratsky A, Steardo L, Parpura V, Montana V. Translational potential of astrocytes in brain disorders. Prog Neurobiol 2016;144:188-205. 10.1016/j.pneurobio.2015.09.003. [PubMed: 26386136]

Vezzani A, Aronica E, Mazarati A, Pittman QJ. Epilepsy and brain inflammation. Exp Neurol 2013;244:11-21. 10.1016/j.expneurol.2011.09.033. [PubMed: 21985866]

Webster CM, et al. Microglial P2Y12 deficiency/inhibition protects against brain ischemia. PLoS One 2013;8:e70927 10.1371/journal.pone.0070927. [PubMed: 23940669]

Woolf A, Wright R, Amarasiriwardena C, Bellinger D. A child with chronic manganese exposure from drinking water. Environ Health Perspect 2002;110:613-6.

Wyss-Coray T, Mucke L. Inflammation in neurodegenerative disease--a double-edged sword. Neuron 2002;35:419-32. [PubMed: 12165466]

Xu B, Xu ZF, Deng Y. Protective effects of MK-801 on manganese-induced glutamate metabolism disorder in rat striatum. Exp Toxicol Pathol 2010;62:381-90. 10.1016/j.etp.2009.05.007. [PubMed: 19540097]

Yin Z, et al. Methylmercury induces oxidative injury, alterations in permeability and glutamine transport in cultured astrocytes. Brain Res 2007;1131:1-10. 10.1016/j.brainres.2006.10.070. [PubMed: 17182013]

Zhang S, Zhou Z, Fu J. Effect of manganese chloride exposure on liver and brain mitochondria function in rats. Environ Res 2003;93:149-57. [PubMed: 12963399]

Zhang W, et al. Aggregated alpha-synuclein activates microglia: a process leading to disease progression in Parkinson's disease. FASEB J 2005;19:533-42. 10.1096/fj.04-2751com. [PubMed: 15791003]

Zhang P, et al. Microglia enhance manganese chloride-induced dopaminergic neurodegeneration: role of free radical generation. Exp Neurol 2009;217:219-30. 10.1016/j.expneurol.2009.02.013. [PubMed: 19268665]

Zhang P, Lokuta KM, Turner DE, Liu B. Synergistic dopaminergic neurotoxicity of manganese and lipopolysaccharide: differential involvement of microglia and astroglia. J Neurochem 2010;112:434-43. 10.1111/j.1471-4159.2009.06477.x. [PubMed: 19895668] 
Zhao F, et al. Manganese induces dopaminergic neurodegeneration via microglial activation in a rat model of manganism. Toxicol Sci 2009;107:156-64. 10.1093/toxsci/kfn213. [PubMed:

18836210] 